\title{
Reformulation of the Stochastic Potential Switching Algorithm and a Generalized Fourtuin-Kasteleyn Representation
}

\author{
Munetaka Sasaki \\ Department of Applied Physics, Tohoku University, Sendai, 980-8579
}

(Dated: November 16, 2018)

\begin{abstract}
A new formulation of the stochastic potential switching algorithm is presented. This reformulation naturally leads us to a generalized Fourtuin-Kasteleyn representation of the partition function $Z$. A formula for internal energy $E$ and that of heat capacity $C$ are derived from derivatives of the partition function. We also derive a formula for the exchange probability in the replica exchange Monte Carlo method. By combining the formulae with the Stochastic Cutoff method, we can greatly reduce the computational time to perform internal energy and heat capacity measurements and the replica exchange Monte Carlo method in long-range interacting systems. Numerical simulations in three dimensional magnetic dipolar systems show the validity of the method.

PACS numbers: 02.70.Tt, 02.70.Rr, 05.10.Ln, 75.10.Hk
\end{abstract}

\section{INTRODUCTION}

One of the most challenging subjects in the field of computational physics is to develop efficient methods for long-range interacting systems. The difficulty of longrange interacting systems comes from the fact that the number of interactions rapidly increases with increasing the number of elements $N$ of the system. For example, in the case of systems with pairwise interactions, the number of interactions is proportional to $N^{2}$. Therefore, if one uses a naive simulation method in such systems, the computational time per one step rapidly increases in proportion to $N^{2}$, which is quite contrast to the case of short-range interacting systems in which the computational time increases in proportion to $N$. In order to overcome the difficulty, many simulation methods have been proposed until now [1 10 .

Recently, the author and Matsubara have developed an efficient Monte Carlo (MC) method called Stochastic CutOff (SCO) method for long-range interacting systems [11]. The basic idea of the method is to switch long-range interactions $V_{i j}$ stochastically to either zero or a pseudointeraction $\bar{V}_{i j}$ with the detailed balance condition satisfied. Interactions are switched by using the Stochastic Potential Switching (SPS) algorithm 12, 13]. Because most of the distant and weak interactions are eliminated by being switched to zero, the SCO method greatly reduces the number of interactions and computational time in long-range interacting systems [14]. Furthermore, this method does not involve any approximation because the detailed balance condition is satisfied strictly. Fukui and Todo have recently developed an efficient MC method based on similar strategy by use of different pseudointeractions and different way of switching interactions [15].

In the present work, we reformulate the SPS algorithm which is used in the SCO method. This reformulation gives us a generalized version of the Fourtuin-Kasteleyn representation of the partition function in the Ising ferromagnetic model [16, 17]. This representation of the partition function is used to derive new formulae for in- ternal energy and heat capacity measurements. Since these formulae consist of only terms which survive as $\bar{V}_{i j}$ in the above-mentioned potential switching process, the computational time for the measurements are reduced greatly. We also derive an formula which reduces the computational time to estimate exchange probability in the replica exchange MC method [18]. In order to verify the formulae, we perform some MC simulations in three dimensional magnetic dipolar systems. The results clearly show the validity of the formulae.

The organization of the paper is as follows. In \$II we reformulate the stochastic potential switching algorithm. A generalized Fourtuin-Kasteleyn representation of the partition function is presented in this section. In and $₫ \mathrm{IV}$, we derive formulae for internal energy and heat capacity measurements and a formula for the replica exchange MC method, respectively. The validity of these formulae is confirmed numerically in $\$ \nabla 1$. Section VII is devoted to a summary and discussions.

\section{REFORMULATION OF THE SPS ALGORITHM}

Before reformulating the SPS algorithm, we briefly explain the SPS algorithm [12, 13]. We hereafter consider a system with pairwise long-range interactions described by the Hamiltonian

$$
\mathcal{H}=\sum_{i<j} V_{i j}\left(\boldsymbol{S}_{i}, \boldsymbol{S}_{j}\right)
$$

where $\boldsymbol{S}_{i}$ is a variable associated with the $i$-th element of the system. In this algorithm, $V_{i j}$ is stochastically switched to either $\tilde{V}_{i j}$ or $\bar{V}_{i j}$ with a probability of $P_{i j}$ or $1-P_{i j}$, respectively. The probability $P_{i j}$ is

$$
P_{i j}\left(\boldsymbol{S}_{i}, \boldsymbol{S}_{j}\right)=\exp \left[\beta\left(\Delta V_{i j}\left(\boldsymbol{S}_{i}, \boldsymbol{S}_{j}\right)-\Delta V_{i j}^{*}\right)\right],
$$

where $\beta$ is the inverse temperature,

$$
\Delta V_{i j}\left(\boldsymbol{S}_{i}, \boldsymbol{S}_{j}\right) \equiv V_{i j}\left(\boldsymbol{S}_{i}, \boldsymbol{S}_{j}\right)-\tilde{V}_{i j}\left(\boldsymbol{S}_{i}, \boldsymbol{S}_{j}\right),
$$


and $\Delta V_{i j}^{*}$ is a constant equal to (or greater than) the maximum value of $\Delta V_{i j}\left(\boldsymbol{S}_{i}, \boldsymbol{S}_{j}\right)$ over all $\boldsymbol{S}_{i}$ and $\boldsymbol{S}_{j}$. We can choose the potential $\tilde{V}_{i j}$ arbitrarily. On the other hand, using $P_{i j}\left(\boldsymbol{S}_{i}, \boldsymbol{S}_{j}\right)$, the potential $\bar{V}_{i j}$ is given as

$$
\bar{V}_{i j}\left(\boldsymbol{S}_{i}, \boldsymbol{S}_{j}\right)=V_{i j}\left(\boldsymbol{S}_{i}, \boldsymbol{S}_{j}\right)-\beta^{-1} \log \left[1-P_{i j}\left(\boldsymbol{S}_{i}, \boldsymbol{S}_{j}\right)\right] .
$$

With this potential switching process, the algorithm proceeds as follows:

(A) Potentials $V_{i j}$ are switched to either $\tilde{V}_{i j}$ or $\bar{V}_{i j}$ with the probability of $P_{i j}$ or $1-P_{i j}$, respectively.

(B) A standard MC simulation is performed with the switched Hamiltonian $\mathcal{H}^{\prime}$ expressed as

$$
\mathcal{H}^{\prime}=\sum^{\prime} \tilde{V}_{i j}\left(\boldsymbol{S}_{i}, \boldsymbol{S}_{j}\right)+\sum^{\prime \prime} \bar{V}_{i j}\left(\boldsymbol{S}_{i}, \boldsymbol{S}_{j}\right),
$$

where $\sum^{\prime}$ runs over all the potentials switched to $\tilde{V}$ and $\sum^{\prime \prime}$ runs over those switched to $\bar{V}$. The potential is fixed during the simulation.

(C) Return to (A).

It is shown that this MC procedure strictly satisfies the detailed balance condition with respect to the original Hamiltonian of eq. (1).

In the SCO method, $\tilde{V}_{i j}$ is set to zero to reduce the computational time of $\mathcal{H}^{\prime}$ defined by eq. (5). Furthermore, the use of an efficient method enables us to reduce the computational time of the potential switching procedure (A) greatly (see ref. 11] for details).

In order to reformulate the SPS algorithm, we first introduce a graph variable $g_{i j}$ defined by

$$
g_{i j}= \begin{cases}0 & \text { if } V_{i j} \text { is switched to } \tilde{V}_{i j}, \\ 1 & \text { if } V_{i j} \text { is switched to } \bar{V}_{i j} .\end{cases}
$$

We next introduce a weight $\omega\left(\boldsymbol{S}_{i}, \boldsymbol{S}_{j} ; g_{i j}\right)$ defined by

$$
\omega_{i j}\left(\boldsymbol{S}_{i}, \boldsymbol{S}_{j} ; g_{i j}\right)=\left\{\begin{array}{cc}
\mathrm{e}^{-\beta\left\{\tilde{V}_{i j}\left(\boldsymbol{S}_{i}, \boldsymbol{S}_{j}\right)+\Delta V_{i j}^{*}\right\}} & \left(g_{i j}=0\right), \\
\mathrm{e}^{-\beta \bar{V}_{i j}\left(\boldsymbol{S}_{i}, \boldsymbol{S}_{j}\right)} & \left(g_{i j}=1\right) .
\end{array}\right.
$$

This weight is analogous to the weight introduced by Edwards and Sokal [19]. We hereafter show that the SPS algorithm is a MC method which realizes equilibrium distribution defined by

$$
P_{\mathrm{SPS}}\left(\left\{\boldsymbol{S}_{i}\right\},\left\{g_{i j}\right\}\right) \equiv Z_{\mathrm{SPS}}^{-1} \prod_{i<j} \omega_{i j}\left(\boldsymbol{S}_{i}, \boldsymbol{S}_{j} ; g_{i j}\right),
$$

where

$$
Z_{\mathrm{SPS}} \equiv \operatorname{Tr}_{\left\{\boldsymbol{S}_{i}\right\},\left\{g_{i j}\right\}} \prod_{i<j} \omega_{i j}\left(\boldsymbol{S}_{i}, \boldsymbol{S}_{j} ; g_{i j}\right)
$$

As it is readily derived from eqs. (2), (3), (4), and (7), the weight $\omega_{i j}\left(\boldsymbol{S}_{i}, \boldsymbol{S}_{j} ; g_{i j}\right)$ has the following property:

$$
\operatorname{Tr}_{g_{i j}=0,1} \omega_{i j}\left(\boldsymbol{S}_{i}, \boldsymbol{S}_{j} ; g_{i j}\right)=\exp \left[-\beta V_{i j}\left(\boldsymbol{S}_{i}, \boldsymbol{S}_{j}\right)\right]
$$

This equation naturally leads us to the following new representation of the partition function

$$
Z(\beta)=Z_{\mathrm{SPS}}(\beta)=\operatorname{Tr}_{\left\{\boldsymbol{S}_{i}\right\},\left\{g_{i j}\right\}} \prod_{i<j} \omega_{i j}\left(\boldsymbol{S}_{i}, \boldsymbol{S}_{j} ; g_{i j}\right) .
$$

We also find that the probability that some configuration $\left\{\boldsymbol{S}_{i}\right\}$ is realized in the SPS algorithm is given as

$$
P\left(\left\{\boldsymbol{S}_{i}\right\}\right)=\operatorname{Tr}_{\left\{g_{i j}\right\}} P_{\mathrm{SPS}}\left(\left\{\boldsymbol{S}_{i}\right\},\left\{g_{i j}\right\}\right)=P_{\mathrm{B}}\left(\left\{\boldsymbol{S}_{i}\right\}\right),
$$

where $P_{\mathrm{B}}$ is the Boltzmann distribution defined as

$$
P_{\mathrm{B}}\left(\left\{\boldsymbol{S}_{i}\right\}\right)=Z(\beta)^{-1} \exp \left[-\beta \sum_{i<j} V_{i j}\left(\boldsymbol{S}_{i}, \boldsymbol{S}_{j}\right)\right] .
$$

This is the reason why the Boltzmann sampling is achieved by the SPS algorithm.

We next show that the equilibrium distribution of the SPS algorithm is given by eq. (8). Let us denote the transition probability in the step (A) of the SPS algorithm as $W_{\mathrm{A}}\left(\left\{g_{i j}\right\} \rightarrow\left\{g_{i j}^{\prime}\right\} \mid\left\{\boldsymbol{S}_{i}\right\}\right)$ and that in the step (B) as $W_{\mathrm{B}}\left(\left\{\boldsymbol{S}_{i}\right\} \rightarrow\left\{\boldsymbol{S}_{i}^{\prime}\right\} \mid\left\{g_{i j}\right\}\right)$. It should be noted that the procedure in the step (A) updates the graph variables $\left\{g_{i j}\right\}$ with fixing the configuration variables $\left\{\boldsymbol{S}_{i}\right\}$, and that in the step (B) updates $\left\{\boldsymbol{S}_{i}\right\}$ with fixing $\left\{g_{i j}\right\}$. In the following, we will show that the two transition probabilities satisfy the detailed balance conditions

$$
\begin{aligned}
& P_{\mathrm{SPS}}\left(\left\{\boldsymbol{S}_{i}\right\},\left\{g_{i j}\right\}\right) W_{\mathrm{A}}\left(\left\{g_{i j}\right\} \rightarrow\left\{g_{i j}^{\prime}\right\} \mid\left\{\boldsymbol{S}_{i}\right\}\right) \\
& =P_{\mathrm{SPS}}\left(\left\{\boldsymbol{S}_{i}\right\},\left\{g_{i j}^{\prime}\right\}\right) W_{\mathrm{A}}\left(\left\{g_{i j}^{\prime}\right\} \rightarrow\left\{g_{i j}\right\} \mid\left\{\boldsymbol{S}_{i}\right\}\right), \\
& P_{\mathrm{SPS}}\left(\left\{\boldsymbol{S}_{i}\right\},\left\{g_{i j}\right\}\right) W_{\mathrm{B}}\left(\left\{\boldsymbol{S}_{i}\right\} \rightarrow\left\{\boldsymbol{S}_{i}^{\prime}\right\} \mid\left\{g_{i j}\right\}\right) \\
& =P_{\mathrm{SPS}}\left(\left\{\boldsymbol{S}_{i}^{\prime}\right\},\left\{g_{i j}\right\}\right) W_{\mathrm{B}}\left(\left\{\boldsymbol{S}_{i}^{\prime}\right\} \rightarrow\left\{\boldsymbol{S}_{i}\right\} \mid\left\{g_{i j}\right\}\right) .
\end{aligned}
$$

These two equations clearly show that the equilibrium distribution of the SPS algorithm is $P_{\text {SPS }}\left(\left\{\boldsymbol{S}_{i}\right\},\left\{g_{i j}\right\}\right)$.

We start from the proof of eq. (14). It can be easily seen from the procedure in the step $(\mathrm{A})$ that

$$
\begin{aligned}
& W_{\mathrm{A}}\left(\left\{g_{i j}\right\} \rightarrow\left\{g_{i j}^{\prime}\right\} \mid\left\{\boldsymbol{S}_{i}\right\}\right) \\
& =\prod^{(0)^{\prime}} P_{i j}\left(\boldsymbol{S}_{i}, \boldsymbol{S}_{j}\right) \prod^{(1)^{\prime}}\left[1-P_{i j}\left(\boldsymbol{S}_{i}, \boldsymbol{S}_{j}\right)\right]
\end{aligned}
$$

where the product $\prod^{(0)^{\prime}}$ runs over all the pairs with $g_{i j}^{\prime}=$ 0 and $\prod^{(1)^{\prime}}$ runs over those with $g_{i j}^{\prime}=1$. It should be noted that $W_{\mathrm{A}}$ does not depend on $\left\{g_{i j}\right\}$. To rewrite the right hand side of the above equation, we utilize the following two equations: Firstly, it is found from eqs. (2), (3), and (7) that

$$
P_{i j}\left(\boldsymbol{S}_{i}, \boldsymbol{S}_{j}\right)=\omega_{i j}\left(\boldsymbol{S}_{i}, \boldsymbol{S}_{j} ; g_{i j}^{\prime}=0\right) \mathrm{e}^{\beta V_{i j}\left(\boldsymbol{S}_{i}, \boldsymbol{S}_{j}\right)} .
$$

Secondly, we can rewrite $1-P_{i j}\left(\boldsymbol{S}_{i}, \boldsymbol{S}_{j}\right)$ as

$$
\begin{aligned}
& 1-P_{i j}\left(\boldsymbol{S}_{i}, \boldsymbol{S}_{j}\right) \\
& =\left\{\mathrm{e}^{-\beta V_{i j}\left(\boldsymbol{S}_{i}, \boldsymbol{S}_{j}\right)}-\omega_{i j}\left(\boldsymbol{S}_{i}, \boldsymbol{S}_{j} ; g_{i j}^{\prime}=0\right)\right\} \mathrm{e}^{\beta V_{i j}\left(\boldsymbol{S}_{i}, \boldsymbol{S}_{j}\right)} \\
& =\omega_{i j}\left(\boldsymbol{S}_{i}, \boldsymbol{S}_{j} ; g_{i j}^{\prime}=1\right) \mathrm{e}^{\beta V_{i j}\left(\boldsymbol{S}_{i}, \boldsymbol{S}_{j}\right)}
\end{aligned}
$$


where we have used eqs. (10) and (17). By substituting these two equations into eq. (16), we obtain

$$
\begin{aligned}
& W_{\mathrm{A}}\left(\left\{g_{i j}\right\} \rightarrow\left\{g_{i j}^{\prime}\right\} \mid\left\{\boldsymbol{S}_{i}\right\}\right) \\
& =\prod_{i<j} \omega_{i j}\left(\boldsymbol{S}_{i}, \boldsymbol{S}_{j} ; g_{i j}^{\prime}\right) \mathrm{e}^{\beta V_{i j}\left(\boldsymbol{S}_{i}, \boldsymbol{S}_{j}\right)} \\
& =Z_{\mathrm{SPS}} P_{\mathrm{SPS}}\left(\left\{\boldsymbol{S}_{i}\right\},\left\{g_{i j}^{\prime}\right\}\right) \prod_{i<j} \mathrm{e}^{\beta V_{i j}\left(\boldsymbol{S}_{i}, \boldsymbol{S}_{j}\right)} .
\end{aligned}
$$

This equation shows that $W_{\mathrm{A}}$ satisfies the detailed balance condition (14).

Our second task is to prove eq. (15). Since we perform a MC simulation with the switched Hamiltonian $\mathcal{H}^{\prime}$, the transition probability $W_{\mathrm{B}}$ satisfies the detailed balance condition

$$
\begin{aligned}
& \mathrm{e}^{-\beta\left\{\sum^{(0)} \tilde{V}_{i j}\left(\boldsymbol{S}_{i}, \boldsymbol{S}_{j}\right)+\sum^{(1)} \bar{V}_{i j}\left(\boldsymbol{S}_{i}, \boldsymbol{S}_{j}\right)\right\}} \\
& \times W_{\mathrm{B}}\left(\left\{\boldsymbol{S}_{i}\right\} \rightarrow\left\{\boldsymbol{S}_{i}^{\prime}\right\} \mid\left\{g_{i j}\right\}\right) \\
& =\mathrm{e}^{-\beta\left\{\sum^{(0)} \tilde{V}_{i j}\left(\boldsymbol{S}_{i}^{\prime}, \boldsymbol{S}_{j}^{\prime}\right)+\sum^{(1)} \bar{V}_{i j}\left(\boldsymbol{S}_{i}^{\prime}, \boldsymbol{S}_{j}^{\prime}\right)\right\}} \\
& \times W_{\mathrm{B}}\left(\left\{\boldsymbol{S}_{i}^{\prime}\right\} \rightarrow\left\{\boldsymbol{S}_{i}\right\} \mid\left\{g_{i j}\right\}\right),
\end{aligned}
$$

where the sum $\sum^{(0)}$ runs over all the pairs with $g_{i j}=0$ and $\sum^{(1)}$ runs over those with $g_{i j}=1$. By multiplying $\mathrm{e}^{-\beta \sum^{(1)} \Delta V_{i j}^{*}}$ to the both hand sides of the equation, we obtain

$$
\begin{aligned}
& W_{\mathrm{B}}\left(\left\{\boldsymbol{S}_{i}\right\} \rightarrow\left\{\boldsymbol{S}_{i}^{\prime}\right\} \mid\left\{g_{i j}\right\}\right) \prod_{i<j} \omega_{i j}\left(\boldsymbol{S}_{i}, \boldsymbol{S}_{j} ; g_{i j}\right) \\
& =W_{\mathrm{B}}\left(\left\{\boldsymbol{S}_{i}^{\prime}\right\} \rightarrow\left\{\boldsymbol{S}_{i}\right\} \mid\left\{g_{i j}\right\}\right) \prod_{i<j} \omega_{i j}\left(\boldsymbol{S}_{i}^{\prime}, \boldsymbol{S}_{j}^{\prime} ; g_{i j}\right),
\end{aligned}
$$

where we have used eq. (7). It is clear from eq. (8) that this equation is equivalent to the detailed balance condition (15).

We next turn to the new representation of the partition function, i.e., eq. (11). This is a generalization of the Fourtuin-Kasteleyn representation of the partition function in the Ising ferromagnetic model [16, 17]. In fact, it is shown in the appendix A that the original FourtuinKasteleyn representation is derived from eq. (11). This representation is more comprehensive than the original one in the following two points:

1) In the new representation, potential $\tilde{V}_{i j}$ can be chosen arbitrarily. On the other hand, the original Fourtuin-Kasteleyn representation corresponds to a special case in which $\tilde{V}_{i j}$ is zero.

2) The new representation is valid no matter whether the configuration variables $\left\{\boldsymbol{S}_{i}\right\}$ are discrete or continuous. This is contrast to the original representation which is derived for systems with discrete variables.

This generalized Fourtuin-Kasteleyn representation will be used in the next section to derive formulae for internal energy and heat capacity measurements.

\section{FORMULAE FOR INTERNAL ENERGY AND HEAT CAPACITY}

In order to derive formulae for internal energy $\langle E\rangle$ and heat capacity $\langle C\rangle$, we use the following thermodynamic relations:

$$
\langle E\rangle=-Z^{-1} \frac{\partial Z}{\partial \beta},
$$

$$
\langle C\rangle=k_{\mathrm{B}} \beta^{2}\left(Z^{-1} \frac{\partial^{2} Z}{\partial \beta^{2}}-Z^{-2}\left(\frac{\partial Z}{\partial \beta}\right)^{2}\right) .
$$

As shown in the Appendix B, the formulae are obtained by substituting our generalized Fourtuin-Kasteleyn representation eq. (11) into these equations and calculating the derivatives. The results are

$$
\langle E\rangle=E_{\mathrm{const}}+\langle\tilde{E}\rangle_{\mathrm{SPS}},
$$

$$
\langle C\rangle=k_{\mathrm{B}} \beta^{2}\left(\left\langle\tilde{E}^{2}\right\rangle_{\mathrm{SPS}}-\langle\tilde{E}\rangle_{\mathrm{SPS}}^{2}-\left\langle\frac{\partial \tilde{E}}{\partial \beta}\right\rangle_{\mathrm{SPS}}\right),
$$

where

$$
\begin{gathered}
\langle\mathcal{O}\rangle_{\mathrm{SPS}} \equiv \operatorname{Tr}_{\left\{g_{i j}\right\},\left\{\boldsymbol{S}_{i}\right\}} \mathcal{O} P_{\mathrm{SPS}}\left(\left\{\boldsymbol{S}_{i}\right\},\left\{g_{i j}\right\}\right), \\
E_{\text {const }}=\sum_{k<l} \Delta V_{k l}^{*}, \\
\tilde{E}=\sum_{k<l}\left\{\tilde{V}_{k l}+\delta_{g_{k l}, 1}\left(\frac{\Delta V_{k l}-\Delta V_{k l}^{*}}{1-P_{k l}}\right)\right\}, \\
\frac{\partial \tilde{E}}{\partial \beta}=\sum_{k<l} \delta_{g_{k l}, 1}\left(\frac{\Delta V_{k l}-\Delta V_{k l}^{*}}{1-P_{k l}}\right)^{2} P_{k l} .
\end{gathered}
$$

$P_{k l}$ in eqs. (28) and (29) is the switching probability defined by eq. (2). It is quite important to note that the average in $\mathrm{MC}$ simulations is equivalent to $\langle\mathcal{O}\rangle_{\mathrm{SPS}}$, i.e.,

$$
\langle\mathcal{O}\rangle_{\mathrm{MC}}=\langle\mathcal{O}\rangle_{\mathrm{SPS}}
$$

This comes from the fact that MC simulation with the SPS algorithm samples states with the probability $P_{\mathrm{SPS}}\left(\left\{\boldsymbol{S}_{i}\right\},\left\{g_{i j}\right\}\right)$.

In the case of $\tilde{V}_{i j}=0, E_{\text {const }}, \tilde{E}$, and $\frac{\partial \tilde{E}}{\partial \beta}$ in eqs. (27), (28), and (29) are reduced to the following forms:

$$
E_{\text {const }} \equiv \sum_{k<l} V_{k l}^{*}
$$

$$
\tilde{E} \equiv \sum_{k<l} \delta_{g_{k l}, 1}\left(\frac{V_{k l}-V_{k l}^{*}}{1-P_{k l}}\right)
$$




$$
\frac{\partial \tilde{E}}{\partial \beta}=\sum_{k<l} \delta_{g_{k l}, 1}\left(\frac{V_{k l}-V_{k l}^{*}}{1-P_{k l}}\right)^{2} P_{k l}
$$

where $V_{i j}^{*}$ is a constant equal to (or greater than) the maximum value of $V_{i j}\left(\boldsymbol{S}_{i}, \boldsymbol{S}_{j}\right)$ over all $\boldsymbol{S}_{i}$ and $\boldsymbol{S}_{j}$. The point of the formulae is the presence of $\delta_{g_{k l}, 1}$ in $\tilde{E}$ and $\frac{\partial \tilde{E}}{\partial \beta}$. In general, the strength of pairwise long-range interactions $V_{i j}$ decreases with increasing the distance $r_{i j}$. Therefore, $g_{i j}$ for most of distant and weak interactions becomes zero (see eqs. (2) and (6)). This means that the computational time of $\tilde{E}$ and its derivative is much shorter than that of $\sum_{k<l} V_{k l}$ which is needed in naive internal energy and heat capacity measurements. Although the calculation of the constant $E_{\text {const }}$ requires $\mathcal{O}\left(N^{2}\right)$ computational time, it is enough to calculate it just once at the beginning of the simulation.

In summary, the use of the formulae (eqs. (24), (25), (31), (32), and (33)) enables us to reduce the computational time for internal energy and heat capacity measurements greatly. This method can be used in the SCO method [11] because $\tilde{V}_{i j}$ is set to be zero in the method.

\section{A FORMULA FOR THE REPLICA EXCHANGE MC METHOD}

We first explain the replica exchange MC method [18] briefly. This method is quite efficient for systems with rugged energy landscape such as spin glasses. In this method, we consider a system with $M$ independent replicas and $M$ different temperatures. The $M$ replicas have a common Hamiltonian $\mathcal{H}(\boldsymbol{S})$. The purpose of the method is to sample states of the $M$ replica system with the following equilibrium distribution

$$
\mathcal{Q}_{\mathrm{B}}\left(\boldsymbol{S}_{1}, \beta_{1} ; \cdots ; \boldsymbol{S}_{M}, \beta_{M}\right)=\prod_{m=1}^{M} P_{\mathrm{B}}\left(\boldsymbol{S}_{m}, \beta_{m}\right)
$$

where $\boldsymbol{S}_{m}$ denotes the set of real variables $\left\{\boldsymbol{S}_{i}\right\}$ of the $m$-th replica and $P_{\mathrm{B}}$ is the Boltzmann distribution defined by eq. (13). In the replica exchange MC method, the equilibration is accelerated by exchanging the replica at temperature $T_{m}$ for that at $T_{n}$. By employing the Metropolis method, the probability of accepting the exchange is given as

$$
\begin{aligned}
& W_{\mathrm{B}}\left(\boldsymbol{S}, \beta_{m} ; \boldsymbol{S}^{\prime}, \beta_{n} \rightarrow \boldsymbol{S}^{\prime}, \beta_{m} ; \boldsymbol{S}, \beta_{n}\right) \\
& =\min \left\{1, \frac{\mathcal{Q}_{\mathrm{B}}\left(\cdots ; \boldsymbol{S}^{\prime}, \beta_{m} ; \cdots ; \boldsymbol{S}, \beta_{n} ; \cdots\right)}{\mathcal{Q}_{\mathrm{B}}\left(\cdots ; \boldsymbol{S}, \beta_{m} ; \cdots ; \boldsymbol{S}^{\prime}, \beta_{n} ; \cdots\right)}\right\} \\
& =\min \left[1, X_{\mathrm{B}}\right],
\end{aligned}
$$

where

$$
X_{\mathrm{B}}=\exp \left[\left(\beta_{m}-\beta_{n}\right)\left(\mathcal{H}(\boldsymbol{S})-\mathcal{H}\left(\boldsymbol{S}^{\prime}\right)\right)\right]
$$

A problem of the replica exchange $\mathrm{MC}$ method in longrange interacting systems is that it costs $\mathcal{O}\left(N^{2}\right)$ computational time to calculate the exchange probability since
$X_{\mathrm{B}}$ in eq. (36) contains $\mathcal{H}$ which consists of $\mathcal{O}\left(N^{2}\right)$ pairwise interactions. In order to overcome the difficulty, we consider a replica exchange MC method whose equilibrium distribution is

$$
\begin{aligned}
& \mathcal{Q}_{\mathrm{SPS}}\left(\boldsymbol{S}_{1}, \boldsymbol{g}_{1}, \beta_{1} ; \cdots ; \boldsymbol{S}_{M}, \boldsymbol{g}_{M}, \beta_{M}\right) \\
& =\prod_{m=1}^{M} P_{\mathrm{SPS}}\left(\boldsymbol{S}_{m}, \boldsymbol{g}_{m}, \beta_{m}\right),
\end{aligned}
$$

where $\boldsymbol{g}_{m}$ denotes the set of graph variables $\left\{g_{i j}\right\}$ of the $m$-th replica and $P_{\text {SPS }}$ is defined by eq. (8). It should be noted that this replica exchange MC method samples configuration $\left(\boldsymbol{S}_{1}, \cdots, \boldsymbol{S}_{M}\right)$ according to the original probability $\mathcal{Q}_{\mathrm{B}}$ since $\mathcal{Q}_{\mathrm{SPS}}$ is related to $\mathcal{Q}_{\mathrm{B}}$ as

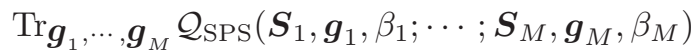

$$
\begin{aligned}
& =\mathcal{Q}_{\mathrm{B}}\left(\boldsymbol{S}_{1}, \beta_{1} ; \cdots ; \boldsymbol{S}_{M}, \beta_{M}\right) \text {, }
\end{aligned}
$$

where we have used eq. (12). The probability of accepting the replica exchange is given as

$$
\begin{aligned}
& W_{\mathrm{SPS}}\left(\boldsymbol{S}, \boldsymbol{g}, \beta_{m} ; \boldsymbol{S}^{\prime}, \boldsymbol{g}^{\prime}, \beta_{n} \rightarrow \boldsymbol{S}^{\prime}, \boldsymbol{g}^{\prime}, \beta_{m} ; \boldsymbol{S}, \boldsymbol{g}, \beta_{n}\right) \\
& =\min \left\{1, \frac{\mathcal{Q}_{\mathrm{SPS}}\left(\cdots ; \boldsymbol{S}^{\prime}, \boldsymbol{g}^{\prime}, \beta_{m} ; \cdots ; \boldsymbol{S}, \boldsymbol{g}, \beta_{n} ; \cdots\right)}{\mathcal{Q}_{\mathrm{SPS}}\left(\cdots ; \boldsymbol{S}, \boldsymbol{g}, \beta_{m} ; \cdots ; \boldsymbol{S}^{\prime}, \boldsymbol{g}^{\prime}, \beta_{n} ; \cdots\right)}\right\} \\
& =\min \left[1, X_{\mathrm{SPS}}\right],
\end{aligned}
$$

where

$$
\begin{aligned}
& X_{\mathrm{SPS}}=\prod^{(0)^{\prime}} \exp \left[\left\{\beta_{n}-\beta_{m}\right\}\left\{\tilde{V}_{i j}\left(\boldsymbol{S}_{i}^{\prime}, \boldsymbol{S}_{j}^{\prime}\right)+\Delta V_{i j}^{*}\right\}\right] \\
& \times \prod^{(0)} \exp \left[\left\{\beta_{m}-\beta_{n}\right\}\left\{\tilde{V}_{i j}\left(\boldsymbol{S}_{i}, \boldsymbol{S}_{j}\right)+\Delta V_{i j}^{*}\right\}\right] \\
& \times \prod^{(1)^{\prime}} \exp \left[\left\{\beta_{n}-\beta_{m}\right\} V_{i j}\left(\boldsymbol{S}_{i}^{\prime}, \boldsymbol{S}_{j}^{\prime}\right)\right] \frac{1-P_{i j}\left(\boldsymbol{S}_{i}^{\prime}, \boldsymbol{S}_{j}^{\prime}, \beta_{m}\right)}{1-P_{i j}\left(\boldsymbol{S}_{i}^{\prime}, \boldsymbol{S}_{j}^{\prime}, \beta_{n}\right)} \\
& \times \prod^{(1)} \exp \left[\left\{\beta_{m}-\beta_{n}\right\} V_{i j}\left(\boldsymbol{S}_{i}, \boldsymbol{S}_{j}\right)\right] \frac{1-P_{i j}\left(\boldsymbol{S}_{i}, \boldsymbol{S}_{j}, \beta_{n}\right)}{1-P_{i j}\left(\boldsymbol{S}_{i}, \boldsymbol{S}_{j}, \beta_{m}\right)} .
\end{aligned}
$$

In this equation, the product $\prod^{(\alpha)}$ runs over all the pairs with $g_{i j}=\alpha$ and $\prod^{(\alpha)^{\prime}}$ runs over those with $g_{i j}^{\prime}=\alpha$.

When $\tilde{V}_{i j}=0$, we can rewrite the first product in the right hand side of eq. (40) as

$$
\begin{aligned}
& \prod^{(0)^{\prime}} \exp \left[\left\{\beta_{n}-\beta_{m}\right\} V_{i j}^{*}\right] \\
& =\prod_{i<j} \exp \left[\left\{\beta_{n}-\beta_{m}\right\} V_{i j}^{*}\right] \prod^{(1)^{\prime}} \exp \left[-\left\{\beta_{n}-\beta_{m}\right\} V_{i j}^{*}\right] .
\end{aligned}
$$

The second product can be rewritten in a similar way. By substituting these results into eq. (40), we find

$$
\begin{aligned}
& X_{\mathrm{SPS}} \\
& =\prod^{(1)^{\prime}} \mathrm{e}^{\left[\left\{\beta_{n}-\beta_{m}\right\}\left\{V_{i j}\left(\boldsymbol{S}_{i}^{\prime}, \boldsymbol{S}_{j}^{\prime}\right)-V_{i j}^{*}\right\}\right]} \frac{1-P_{i j}\left(\boldsymbol{S}_{i}^{\prime}, \boldsymbol{S}_{j}^{\prime}, \beta_{m}\right)}{1-P_{i j}\left(\boldsymbol{S}_{i}^{\prime}, \boldsymbol{S}_{j}^{\prime}, \beta_{n}\right)} \\
& \times \prod^{(1)} \mathrm{e}^{\left[\left\{\beta_{m}-\beta_{n}\right\}\left\{V_{i j}\left(\boldsymbol{S}_{i}, \boldsymbol{S}_{j}\right)-V_{i j}^{*}\right\}\right]} \frac{1-P_{i j}\left(\boldsymbol{S}_{i}, \boldsymbol{S}_{j}, \beta_{n}\right)}{1-P_{i j}\left(\boldsymbol{S}_{i}, \boldsymbol{S}_{j}, \beta_{m}\right)} .
\end{aligned}
$$


Since $X_{\mathrm{SPS}}$ in this formula is calculated only from the pairs whose graph variables are one, it can be calculated with very short computational time as $\tilde{E}^{\prime}$ and $\frac{\partial \tilde{E}^{\prime}}{\partial \beta}$ in eqs. (32) and (33) are.

\section{THE CASE WHEN LONG-RANGE INTERACTIONS AND SHORT-RANGE INTERACTIONS COEXIST}

When long-range interactions $\left\{V_{i j}^{(\mathrm{L})}\right\}$ and short-range interactions $\left\{V_{i j}^{(\mathrm{S})}\right\}$ coexist, we do not need to use the SCO method for short-range interactions because it does not reduce the computational time significantly. In other words, we can switch $V_{i j}^{(\mathrm{L})}$ to 0 or $\bar{V}_{i j}^{(\mathrm{L})}$ with $V_{i j}^{(\mathrm{S})}$ unchanged. This can be realized by setting $\tilde{V}_{i j}^{(\mathrm{L})}=0$ and $\tilde{V}_{i j}^{(\mathrm{S})}=V_{i j}^{(\mathrm{S})}$. In this case, we can decompose $E_{\text {const }}, \tilde{E}$, $\frac{\partial E}{\partial \beta}$, and $X_{\text {SPS }}$ in eqs. (27), (28), (29), and (40) as

$$
\begin{aligned}
& E_{\text {const }}=E_{\text {const }}^{(\mathrm{L})}+E_{\text {const }}^{(\mathrm{S})}, \\
& \tilde{E}=\tilde{E}^{(\mathrm{L})}+\tilde{E}^{(\mathrm{S})}, \\
& \frac{\partial \tilde{E}}{\partial \beta}=\frac{\partial \tilde{E}^{(\mathrm{L})}}{\partial \beta}+\frac{\partial \tilde{E}^{(\mathrm{S})}}{\partial \beta}, \\
& X_{\mathrm{SPS}}=X_{\mathrm{SPS}}^{(\mathrm{L})} \times X_{\mathrm{SPS}}^{(\mathrm{S})},
\end{aligned}
$$

where the first terms and the second terms in the right hand sides denote contributions from long-range interactions and those from short-range interactions, respectively. As we have already mentioned, the first terms are given by eqs. (31), (32), (33), and (42), respectively. On the other hand, by substituting $\tilde{V}_{k l}=V_{k l}^{(\mathrm{S})}$ and $\Delta V_{k l}^{*}=0$ into eqs. (27), (28), (29), and (40), we readily obtain

$E_{\text {const }}^{(\mathrm{S})}=0$,

$\tilde{E}^{(\mathrm{S})}=-\sum_{\langle k l\rangle} V_{k l}^{(\mathrm{S})}$,

$\frac{\partial \tilde{E}^{(\mathrm{S})}}{\partial \beta}=0$,

$X_{\mathrm{SPS}}^{(\mathrm{S})}=\prod_{\langle k l\rangle} \exp \left[\left(\beta_{m}-\beta_{n}\right)\left(V_{k l}\left(\boldsymbol{S}_{k}, \boldsymbol{S}_{l}\right)-V_{k l}\left(\boldsymbol{S}_{k}^{\prime}, \boldsymbol{S}_{l}^{\prime}\right)\right)\right]$ $=X_{\mathrm{B}}^{(\mathrm{S})}$,

where we have used the fact that all the potentials are switched to $\tilde{V}_{k l}$. Note that $P_{k l}=1$ when $\tilde{V}_{k l}=V_{k l}$ and $\Delta V_{i j}^{*}=0$. These results are quite natural because they coincide with the results in the usual MC procedure.

\section{NUMERICAL TESTS}

\section{A. Internal energy and heat capacity measurements}

In order to check the validity of the formulae for internal energy and heat capacity measurements, we perform

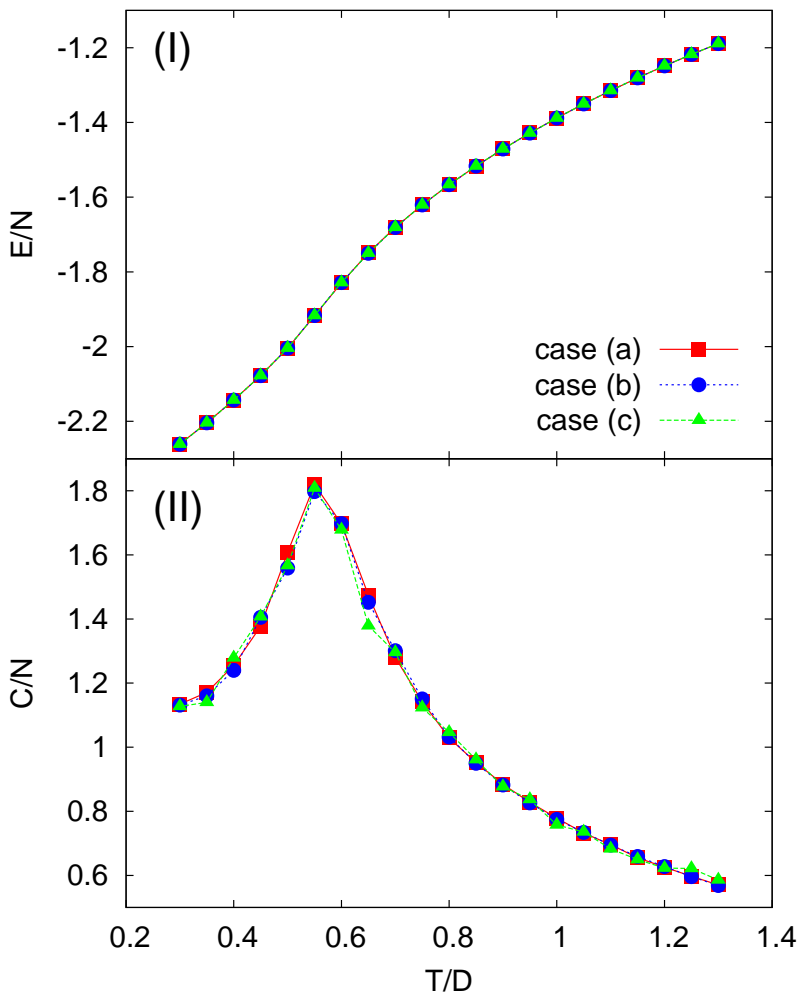

FIG. 1: (Color online) Temperature dependence of (I) internal energy $E / N$ and (II) heat capacity $C / N$ in the purely dipolar system. Simulated annealing method is used for the measurement. The size $N$ is $10^{3}$. Measurements are done in the three cases (see text).

MC simulations of a three dimensional magnetic dipolar system on a $L^{3}$ simple cubic lattice. The boundary condition is open. The Hamiltonian of the system is described as

$$
\mathcal{H}=\mathcal{H}_{\text {dip }}=D \sum_{i<j}\left[\frac{\boldsymbol{S}_{i} \cdot \boldsymbol{S}_{j}-3\left(\boldsymbol{S}_{i} \cdot \boldsymbol{r}_{i j}\right)\left(\boldsymbol{S}_{j} \cdot \boldsymbol{r}_{i j}\right)}{r_{i j}^{3}}\right],
$$

where $\boldsymbol{S}_{i}$ is a classical Heisenberg spin of $\left|\boldsymbol{S}_{i}\right|=1, \boldsymbol{r}_{i j}$ is the vector spanned from a site $i$ to $j$ in the unit of the lattice constant $a, r_{i j}=\left|\boldsymbol{r}_{i j}\right|$, and $D=\left(g \mu_{\mathrm{B}} S\right) / a^{3}$. We hereafter call the system purely dipolar system.

In simulations with the SCO method, we regard each term in the right hand side of eq. (45) as $V_{i j}$. Since $\tilde{V}_{i j}=0$ in the SCO method, $\Delta V_{i j}$ defined by eq. (3) is equal to $V_{i j}$. Interaction $V_{i j}$ has the maximum value $2 D / r_{i j}^{3}$ when $\boldsymbol{S}_{i}$ and $\boldsymbol{S}_{j}$ are anti-parallel along $\boldsymbol{r}_{i j}$. We therefore set $\Delta V_{i j}^{*}$, which should be equal to or greater than $\Delta V_{i j}$ over all $\boldsymbol{S}_{i}$ and $\boldsymbol{S}_{j}$, to $2 D / r_{i j}^{3}$. By substituting these into eq. (2), we obtain

$$
P_{i j}=\exp \left[\beta D\left\{\frac{\boldsymbol{S}_{i} \cdot \boldsymbol{S}_{j}-3\left(\boldsymbol{S}_{i} \cdot \boldsymbol{r}_{i j}\right)\left(\boldsymbol{S}_{j} \cdot \boldsymbol{r}_{i j}\right)-2}{r_{i j}^{3}}\right\}\right],
$$




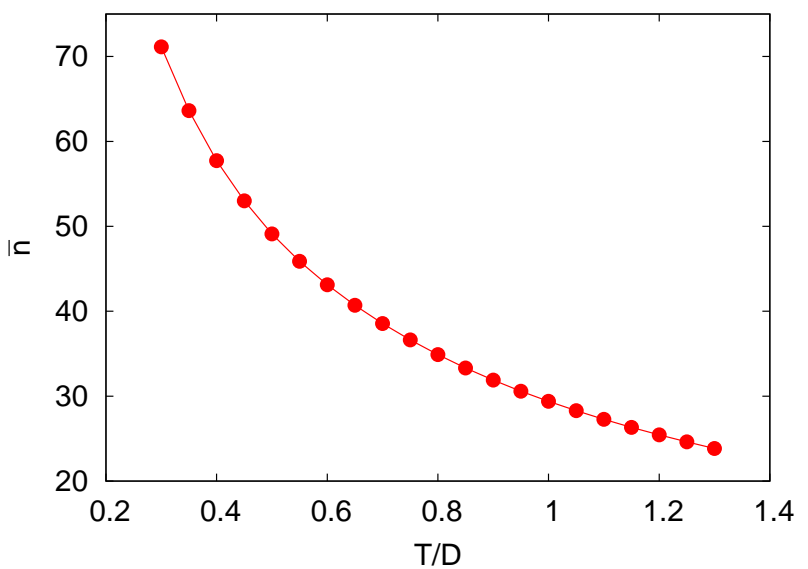

FIG. 2: (Color online) Temperature dependence of the average number $\bar{n}$ of potentials per site which are switched to $\bar{V}_{i j}$.

Pseudointeraction $\bar{V}_{i j}$ is given by substituting $P_{i j}$ of the above equation into eq. (4).

To make a comparison between the new measurement methods and the usual ones, we do simulation in the following three cases:

(a) Usual MC method with usual measurements.

(b) SCO method with usual measurements.

(c) SCO method with measurements by using the formulae derived in $₫$ III.

In all the three cases, simulated annealing method is used. The system is gradually cooled from an initial temperature $T=1.3 D$ to $0.3 D$ in steps of $\Delta T=0.05 D$. The system is kept at each temperature for $2 \times 10^{6} \mathrm{MC}$ steps. The first $10^{6} \mathrm{MC}$ steps are for equilibration and the following $10^{6} \mathrm{MC}$ steps are for measurement. The size $N$ is $10^{3}$. The size is not so large because we do simulation not only with the SCO method but also with a usual method which requires $\mathcal{O}\left(N^{2}\right)$ computational time per one $\mathrm{MC}$ step.

In Fig. 1, internal energy $E$ and heat capacity $C$ measured in the three cases are plotted as a function of temperature. We see that all the data nicely collapse into a single curve, showing the validity of the formulae derived in $₫$ III. We also see that heat capacity has a peak around $T / D=0.55$. This result is consistent with previous works which show the existence of a phase transition around this temperature [20, 21]. Figure 2 shows the average number $\bar{n}$ of potentials per site that survive as $\bar{V}_{i j}$ in the potential switching process. Though $\bar{n}$ increases with decreasing temperature, $\bar{n} \approx 70$ even at the lowest temperature. This means that more than 90 percent of interactions are cut off by being switched to $\tilde{V}_{i j}=0$. It is worth pointing out that the SCO method becomes more efficient with increasing the size. In fact, in the study of two-dimensional magnetic dipolar system with dipolar interactions and ferromagnetic

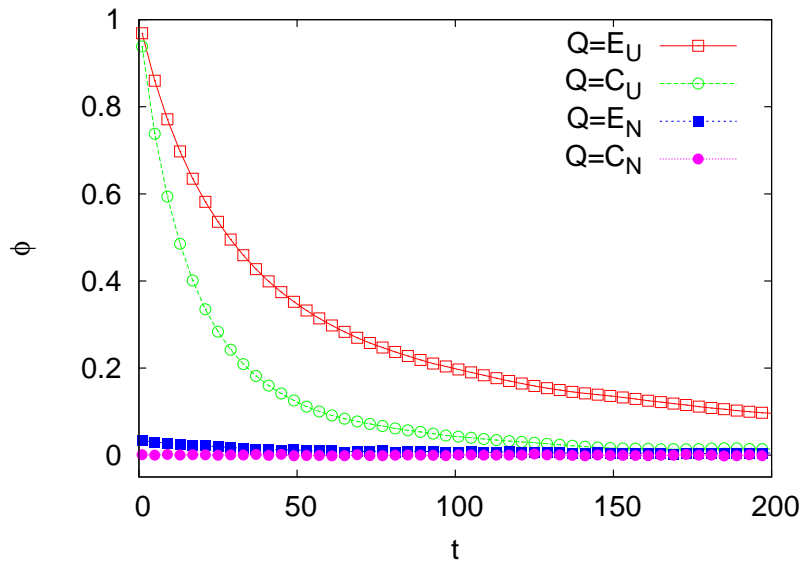

FIG. 3: (Color online) Time autocorrelation functions of the four observables $E_{\mathrm{U}}, C_{\mathrm{U}}, E_{\mathrm{N}}$, and $C_{\mathrm{N}}$ (see eq. (51) for their definitions). The size $N$ is $10^{3}$ and the temperature $T$ is $0.3 D$. All the four observables are measured with the SCO method.

exchange interactions [11], it has been found that the increase of $\bar{n}$ with size is very slow and $\bar{n}$ is 22.5 even when $N=256^{2}=65,536$.

\section{B. Statistical error of the new measurement methods}

We notice from Fig. 1 (II) that the data in the case (c), i.e., those which are measured with eq. (25), fluctuate more than the other data. To get some insights of this behavior, we consider estimating statistical error of observables. We suppose that an observable $Q$ is successively measured $N$ times in a MC simulation to estimate the average $\frac{1}{N} \sum_{\mu=1}^{N} Q_{\mu}$. We assume that the measurement is done every $\delta t$ steps. Although this average value is close to the thermal average value $\langle Q\rangle$, they are slightly different because the number of the measurement $N$ is finite. We hereafter call the difference $\delta Q \equiv \frac{1}{N} \sum_{\mu=1}^{N} Q_{\mu}-\langle Q\rangle$ statistical error. When the period of the measurement $N \delta t$ is much larger than the correlation time $\tau_{Q}$ of the observable $Q$, the expectation value of the square of the statistical error $\left\langle(\delta Q)^{2}\right\rangle$ is approximately evaluated as [22, 23]

$$
\left\langle(\delta Q)^{2}\right\rangle=\frac{1}{N}\left[\left\langle Q^{2}\right\rangle-\langle Q\rangle^{2}\right]\left(1+2 \frac{\tau_{Q}}{\delta t}\right) .
$$

The factor $\left(1+2 \frac{\tau_{Q}}{\delta t}\right)$ in the right hand side of the equation comes from the fact that $Q_{\mu}$ 's measured successively are correlated with each other. From this equation, we can estimate the relative statistical error as

$$
\frac{\sqrt{\left\langle(\delta Q)^{2}\right\rangle}}{\langle Q\rangle}=\frac{C_{\mathrm{SE}}}{\sqrt{N}}
$$


TABLE I: Correlation time $\tau_{Q}$, relative variance, and $C_{\mathrm{SE}}$ of the four observables $E_{\mathrm{U}}, C_{\mathrm{U}}, E_{\mathrm{N}}$, and $C_{\mathrm{N}}$ (see eq. (49) for the definition of $C_{\mathrm{SE}}$ and eq. (51) for the definitions of the four observables). The size $N$ is $10^{3}$ and the temperature $T$ is $0.3 D$. The time interval $\delta t$ in eq. (49) is assumed to be one in the estimation of $C_{\mathrm{SE}}$.

\begin{tabular}{lccc}
\hline \hline & $\tau_{Q}$ & $\frac{\left\langle Q^{2}\right\rangle-\langle Q\rangle^{2}}{\langle Q\rangle^{2}}$ & $C_{\mathrm{SE}}$ \\
\hline$Q=E_{\mathrm{U}}$ & 96 & $2.0 \times 10^{-5}$ & 0.062 \\
$Q=E_{\mathrm{N}}$ & 3.6 & $5.7 \times 10^{-4}$ & 0.068 \\
$Q=C_{\mathrm{U}}$ & 25 & 2.0 & 10 \\
$Q=C_{\mathrm{N}}$ & 0 & $1.6 \times 10^{3}$ & 40 \\
\hline \hline
\end{tabular}

where

$$
C_{\mathrm{SE}}=\sqrt{\frac{\left\langle Q^{2}\right\rangle-\langle Q\rangle^{2}}{\langle Q\rangle^{2}}\left(1+2 \frac{\tau_{Q}}{\delta t}\right)} .
$$

In order to estimate $\tau_{Q}$ in eq. (49), we measure the normalized time autocorrelation function defined by

$$
\phi_{Q}(t) \equiv \frac{\overline{Q(0) Q(t)}-(\bar{Q})^{2}}{\overline{Q^{2}}-(\bar{Q})^{2}},
$$

where $\cdots$ denotes the average over a sequence of the data obtained by a MC simulation. Since we are interested in how correlation times in internal energy and heat capacity measurements are affected by their methods, we calculate correlation functions of the following four observables

$$
\begin{aligned}
& E_{\mathrm{U}}(t)=\mathcal{H}(t), \\
& C_{\mathrm{U}}(t)=k_{\mathrm{B}} \beta^{2}(\mathcal{H}(t)-\langle\mathcal{H}\rangle)^{2}, \\
& E_{\mathrm{N}}(t)=\tilde{E}(t)+E_{\text {const }}, \\
& C_{\mathrm{N}}(t)=k_{\mathrm{B}} \beta^{2}\left\{(\tilde{E}(t)-\langle\tilde{E}\rangle)^{2}-\frac{\partial \tilde{E}(t)}{\partial \beta}\right\},
\end{aligned}
$$

where the subscripts $\mathrm{U}$ and $\mathrm{N}$ denote the usual measurement and the new measurement by using the formulae derived in $\coprod$ III respectively. The results are shown in Fig. 3. All the four observables are measured with the SCO method. We see that correlation functions in new measurements (full symbols) are much smaller than those in usual measurements (open symbols). This probably comes from the fact that $\tilde{E}$ and $\frac{\partial \tilde{E}}{\partial \beta}$ can change without change in spin configurations since they depend on not only $\left\{\boldsymbol{S}_{i}\right\}$ but also $\left\{g_{i j}\right\}$. This result shows that large fluctuations in heat capacity measured with the new formula do not originate from an increase in the correlation time.

We next estimate the correlation time $\tau_{Q}$ from $\phi_{Q}(t)$ as

$$
\tau_{Q}=\int_{0}^{\infty} \mathrm{d} t^{\prime} \phi_{Q}\left(t^{\prime}\right)
$$

In practice, we set the lower limit to be one and adjust the upper limit so that it is much larger than the correlation time. For example, the upper limit was set to 3, 000 when we estimated the correlation time for $E_{\mathrm{U}}$. The estimated values are shown in Table @. We estimate the correlation time for $C_{\mathrm{N}}$ to be zero because it is negligibly small. We calculated $\tau$ for $C_{\mathrm{N}}$ with changing the upper limit from 2 to 10,000 , and found that it does not exceed 0.2 for any upper limits.

In Table I, we also show the values of relative variance and those of $C_{\mathrm{SE}}$. We find that the relative variances in new measurements are larger than those in usual measurements, especially in the heat capacity. Concerning the internal energy, we can explicitly show the increase in variance from eq. (25) as

$$
\begin{aligned}
k_{\mathrm{B}}^{-1} T^{2} C=\left\langle\mathcal{H}^{2}\right\rangle-\langle\mathcal{H}\rangle^{2} & =\left\langle\tilde{E}^{2}\right\rangle-\langle\tilde{E}\rangle^{2}-\left\langle\frac{\partial \tilde{E}}{\partial \beta}\right\rangle \\
& \leq\left\langle\tilde{E}^{2}\right\rangle-\langle\tilde{E}\rangle^{2} \\
& =\left\langle E_{\mathrm{N}}^{2}\right\rangle-\left\langle E_{\mathrm{N}}\right\rangle^{2}
\end{aligned}
$$

where we have used the fact that $\frac{\partial \tilde{E}}{\partial \beta}$ defined by eq. 291 is always positive. Since $\left\langle E_{\mathrm{N}}\right\rangle=\left\langle E_{\mathrm{U}}\right\rangle$, the inequality (53) shows that the relative variance of $E_{\mathrm{N}}$ is larger than that of $E_{\mathrm{U}}$. We next turn to how $C_{\mathrm{SE}}$ is affected by the measurement methods. We see from Table@ that there is no significant difference between $C_{\mathrm{SE}}$ for $E_{\mathrm{U}}$ and that for $E_{\mathrm{N}}$. On the other hand, $C_{\mathrm{SE}}$ for $C_{\mathrm{N}}$ is four times larger than that for $C_{\mathrm{U}}$. This is the reason why the heat capacity in the case (c) fluctuates more than that in the other cases. It should be noted that the relative statistical error is proportional to $C_{\mathrm{SE}}$ (see eq. (48)). Equation (48) also tells us that the number of measurements with $C_{\mathrm{N}}$ should be 16 times as large as that with $C_{\mathrm{U}}$ to make both the statistical errors the same.

In summary, to attain a certain accuracy, estimation of the heat capacity with $C_{\mathrm{N}}$ requires larger number of measurements than that with $C_{\mathrm{U}}$ since fluctuations in $C_{\mathrm{N}}$ are larger than those in $C_{\mathrm{U}}$. On the other hand, the efficiency in the internal energy measurement with $E_{\mathrm{N}}$ is almost the same as that with $E_{\mathrm{U}}$ in the present case. When the heat capacity measurement with $C_{\mathrm{N}}$ does not work well, it might be possible to estimate the heat capacity with lower statistical error by doing numerical differential of the internal energy which is evaluated with $E_{\mathrm{N}}$.

\section{Replica exchange method}

To examine the validity of the formula derived for the replica exchange $\mathrm{MC}$ method, we again do simulations in the following three cases:

(a) Usual MC method with usual replica exchange MC method. 


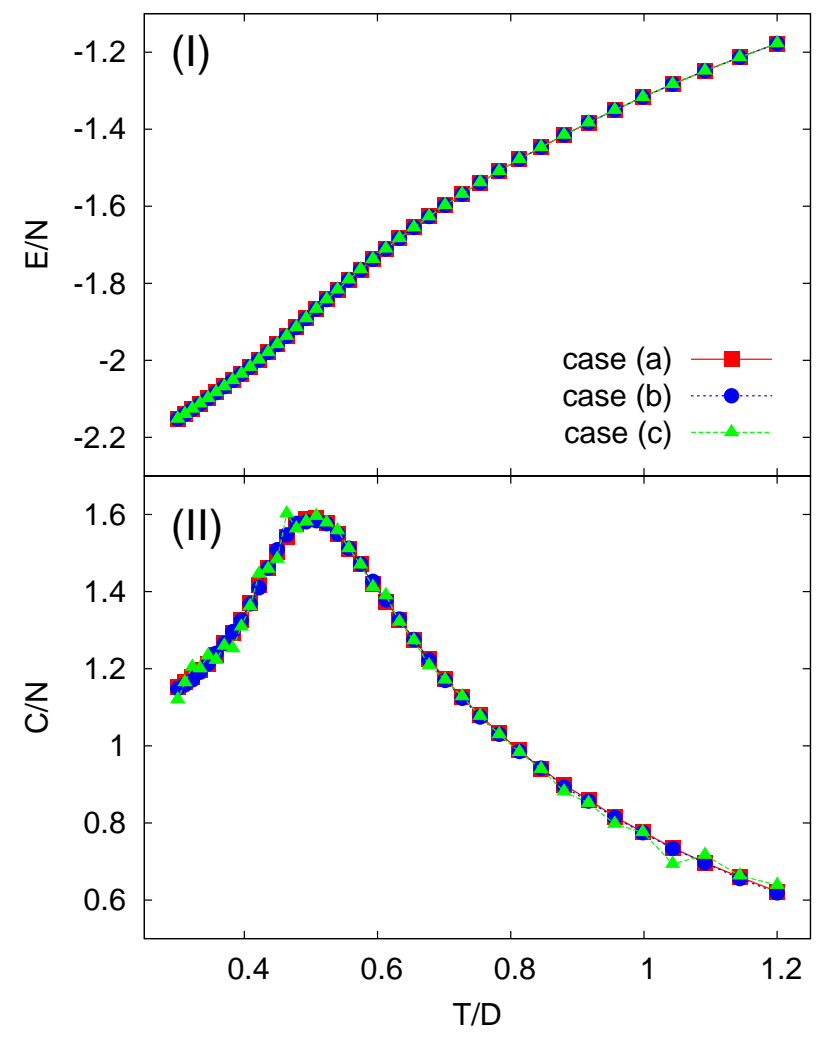

FIG. 4: (Color online) Temperature dependence of (I) internal energy $E / N$ and (II) heat capacity $C / N$ in the purely dipolar system. The replica exchange MC method is used for the measurement. The size $N$ is $6^{3}$. Measurements are done in the three cases (see text).

(b) SCO method with usual replica exchange MC method.

(c) SCO method with replica exchange MC method by using the formula derived in $₫ \mathrm{IV}$.

In all the three cases, the number of MC steps for equilibration and that for measurements are $10^{6}$. The number of temperatures is 40 and the size $N$ is $6^{3}$. Figure 4 shows the result of internal energy and heat capacity measurements. The measurements are done with usual method in the cases (a) and (b), and with our formulae (eqs. (24) and (25)) in the case (c). We again see that all the data nicely collapse into a single curve. This result clearly shows the validity of the formula derived in 1 IV We also see that the heat capacity in the case (c) fluctuates more than that in the other cases, as we

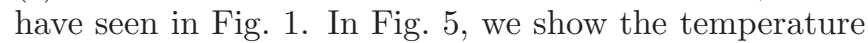
dependence of the probability $P_{\mathrm{ex}}\left(T_{i}\right)$ that exchange trials between $i$-th and $i+1$-th replicas are accepted. We find that $P_{\mathrm{ex}}\left(T_{i}\right)$ with our formula (case (c)) is smaller than that with usual method (the other cases). From this result, one may consider that our replica exchange MC method is less efficient than the usual method. However, this is not true because the computational time of $X_{\mathrm{SPS}}$

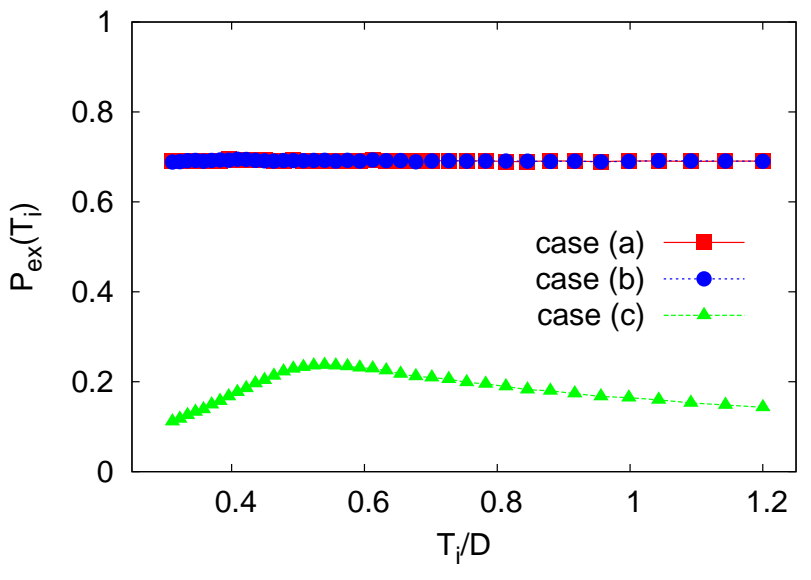

FIG. 5: (Color online) Temperature dependence of the replica exchange probability $P_{\text {ex }}$ in the purely dipolar system. The size $N$ is $6^{3}$ and the number of temperatures is 40 .

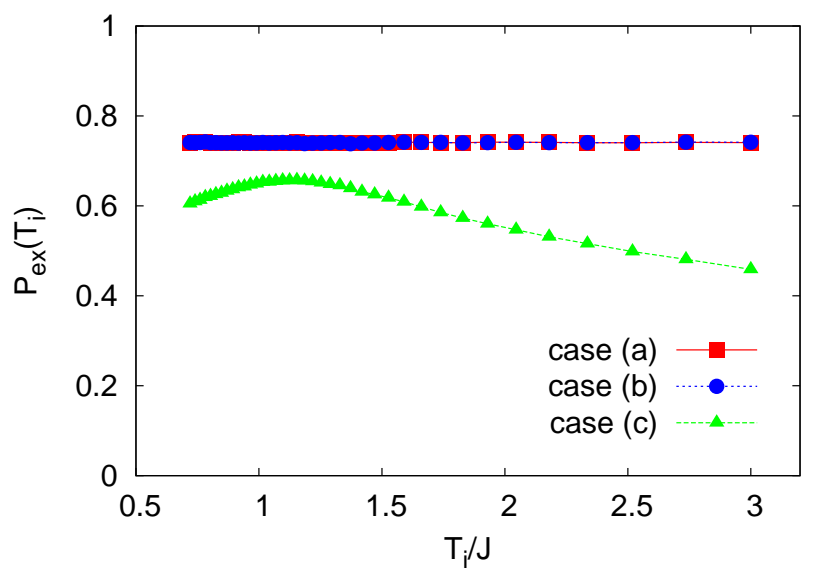

FIG. 6: (Color online) Temperature dependence of the replica exchange probability $P_{\mathrm{ex}}$ in the ferromagnetic dipolar system. The size $N$ is $6^{3}$ and the number of temperatures is 40 .

(eq. (42)) is much shorter than that of $X_{\mathrm{B}}$ (eq. (36)).

We next show results of the replica exchange MC method when long-range interactions and short-range interactions coexist. The Hamiltonian is consist of the long-range magnetic dipolar interactions (eq. (45)) and the short-range exchange interactions

$$
\mathcal{H}_{\mathrm{ex}}=-J \sum_{\langle i j\rangle} \boldsymbol{S}_{i} \cdot \boldsymbol{S}_{j} \quad(J>0),
$$

where the sum runs over the nearest neighbouring pairs. The ratio $D / J$ is 0.1 . We hereafter call the system ferromagnetic dipolar system. The SCO method is used only for the dipolar interactions. We again examine the three cases mentioned in the previous paragraph. The number of $\mathrm{MC}$ steps for equilibration and that for measurements are $10^{6}$. The number of temperatures is 40 and the size $N$ is $6^{3}$. We first have confirmed that internal energy and heat capacity in the three cases coincide with each other. We next examine the temperature dependence of 
TABLE II: Ergod time in the purely dipolar system and the ferromagnetic dipolar system. Measurements are done in the three cases (see text).

\begin{tabular}{lcc}
\hline \hline & purely dipolar & ferromagnetic dipolar \\
\hline case (a) & $1.9 \times 10^{4}$ & $3.3 \times 10^{3}$ \\
case (b) & $4.4 \times 10^{4}$ & $3.5 \times 10^{3}$ \\
case (c) & $6.2 \times 10^{4}$ & $4.5 \times 10^{3}$ \\
\hline \hline
\end{tabular}

the replica exchange probability $P_{\text {ex }}\left(T_{i}\right)$. Figure 6 shows the result. We see that the reduction of the exchange probability in case (c) is not as large as that of the purely dipolar system (Fig. (5). This result is reasonable because the SCO method is used only for the long range interactions and its contribution $\left(X_{\mathrm{SPS}}^{\mathrm{L}}\right.$ in eq. (43) $\left.\left.\mathrm{d}\right)\right)$ is not large. Recall that the ratio $D / J$ is 0.1 . We also measure the ergod time defined by the average MC step for a specific replica to move from the lowest to the highest temperature and return to the lowest one. The result is shown in Table II. In both the systems, the ergod time in the cases (b) and (c) is larger than than that in the case (a), meaning that the use of the SCO method increases the ergod time. However, the increase of the ergod time in the ferromagnetic dipolar system is not as large as that in the purely dipolar system. These results show that the SCO method is particularly efficient for systems with strong short-range interactions and weak long-range interactions. This feature of the SCO method has already been pointed out in the previous work [11].

\section{SUMMARY}

In the present work, we have derived useful formulae for the SCO method to estimate internal energy, heat capacity, and replica exchange probability in the replica exchange MC method. We can reduce the computational time of these quantities greatly by using the formulae because they only contain terms which are not cut off by the $\mathrm{SCO}$ method. On the other hand, we have found that the use of the formulae could cause a decline in the efficiency of the measurement and that in the exchange probability. When the new methods do not work well, the analyses done in the present paper, such as the estimations of the statistical error, the replica exchange probability, and the ergod time, might be helpful to figure out the reason and to get rid of it. Anyway, we hope that these formulae make the SCO method more useful and attractive.

The other achievement of the present work is the derivation of the new Fourtuin-Kasteleyn representation of the partition function, i.e., eq. (11). This representation is more comprehensive than the original one because our representation includes arbitrariness in the choice of pseudointeractions $\left\{\tilde{V}_{i j}\right\}$. Furthermore, this representation can be used no matter whether the variables $\left\{\boldsymbol{S}_{i}\right\}$ are discrete or continuous. We hope that this representa- tion becomes the basis of new algorithms as the original Fourtuin-Kasteleyn representation lead to the SwendsenWang cluster algorithm [24] in the Ising ferromagnetic model.

\section{Acknowledgments}

This work is supported by a Grant-in-Aid for Scientific Research (\#21740279) from MEXT in Japan.

\section{Appendix A: Derivation of the Fourtuin-Kasteleyn representation of the partition function in the Ising ferromagnetic model from eq. (11)}

In this appendix, we consider the Ising ferromagnetic model whose Hamiltonian is described as

$$
\mathcal{H}=-J \sum_{\langle i j\rangle} \sigma_{i} \sigma_{j}
$$

where $\sigma_{i}= \pm 1$ and $J>0$. We set $V_{i j}=-J \sigma_{i} \sigma_{j}$ and consider a special case that $\tilde{V}_{i j}=0$ and $V_{i j}^{*}=J$. Then, we find

$$
P_{i j}=\left\{\begin{array}{cc}
\exp (-2 \beta J) & \left(\sigma_{i}=\sigma_{j}\right) \\
1 & \left(\sigma_{i}=-\sigma_{j}\right)
\end{array}\right.
$$

and

$$
\bar{V}_{i j}=\left\{\begin{array}{cc}
-J-\beta^{-1} \log [1-\exp (-2 \beta J)], & \left(\sigma_{i}=\sigma_{j}\right), \\
\infty & \left(\sigma_{i}=-\sigma_{j}\right) .
\end{array}\right.
$$

By substituting this equation into eq. (7), we obtain

$$
\begin{aligned}
& \omega_{i j}\left(\sigma_{i}, \sigma_{j} ; g_{i j}\right) \\
& =\left\{\begin{array}{cc}
\exp (-\beta J) & \left(g_{i j}=0\right), \\
\exp (\beta J)[1-\exp (-2 \beta J)] \delta_{\sigma_{i}, \sigma_{j}} & \left(g_{i j}=1\right) .
\end{array}\right.
\end{aligned}
$$

It is important to note that the product $\prod_{\langle i j\rangle} \omega_{i j}$ is proportional to the joint distribution of $\left\{\boldsymbol{S}_{i}\right\}$ and $\left\{g_{i j}\right\}$ introduced by Edwards and Sokal [19]. In this sense, we can regard the product of $\omega_{i j}$ defined by eq. (7) as a generalization of Edwards and Sokal's joint distribution.

As it has been pointed out in ref. [19], we can easily obtain the Fourtuin-Kasteleyn representation of the partition function from this joint distribution. We first rewrite $\prod_{\langle i j\rangle} \omega_{i j}$ as

$$
\begin{aligned}
\prod_{\langle i j\rangle} \omega_{i j}= & \mathrm{e}^{N_{\mathrm{int}} \beta J}\{\exp (-2 \beta J)\}^{N_{\mathrm{int}}-N_{\mathrm{b}}} \\
& \times\{1-\exp (-2 \beta J)\}^{N_{\mathrm{b}}} \prod_{\langle i j\rangle}^{(1)} \delta_{\sigma_{i}, \sigma_{j}},
\end{aligned}
$$

where $N_{\text {int }}=\sum_{\langle i j\rangle}$ and $N_{\mathrm{b}}=\sum_{\langle i j\rangle} \delta_{g_{i j}, 1}$. The product $\prod_{\langle i j\rangle}^{(1)}$ runs over all the pairs with $g_{i j}=1$. By using 
eq. (11), we obtain

$$
\begin{aligned}
Z(\beta)= & \mathrm{e}^{N_{\mathrm{int}} \beta J} \operatorname{Tr}_{\left\{g_{i j}\right\}}\{\exp (-2 \beta J)\} N_{\mathrm{int}}-N_{\mathrm{b}} \\
& \times\{1-\exp (-2 \beta J)\}^{N_{\mathrm{b}}} \operatorname{Tr}_{\left\{\sigma_{i}\right\}} \prod_{\langle i j\rangle}^{(1)} \delta_{\sigma_{i}, \sigma_{j}} .
\end{aligned}
$$

We next consider calculating $\operatorname{Tr}_{\left\{\sigma_{i}\right\}} \prod_{\langle i j\rangle}^{(1)} \delta_{\sigma_{i}, \sigma_{j}}$ in the above equation. We hereafter call pairs with $g_{i j}=1$ bonds and a set of sites which are connected by bonds a cluster. Because of the presence of $\delta_{\sigma_{i}, \sigma_{j}}$, the values of $\sigma_{i}$ in a cluster are forced to be the same. Therefore, we find

$$
\operatorname{Tr}_{\left\{\boldsymbol{S}_{i}\right\}} \prod_{\langle i j\rangle}^{(1)} \delta_{\sigma_{i}, \sigma_{j}}=2^{N_{\text {cluster }}\left\{g_{i j}\right\}},
$$

where $N_{\text {cluster }}$ is the number of clusters. By substituting this equation into eq. (A6), we obtain the FourtuinKasteleyn representation of the partition function in the Ising ferromagnetic model, i.e.,

$$
\begin{aligned}
Z(\beta)= & \mathrm{e}^{N_{\text {int }} \beta J} \operatorname{Tr}_{\left\{g_{i j}\right\}}\{\exp (-2 \beta J)\}^{N_{\text {int }}-N_{\mathrm{b}}} \\
& \times\{1-\exp (-2 \beta J)\}^{N_{\mathrm{b}}} \times 2^{N_{\text {cluster }}\left\{g_{i j}\right\}} .
\end{aligned}
$$

\section{Appendix B: Derivation of eqs. (24)-(29)}

We first derive a formula for internal energy $\langle E\rangle$. We see from eqs. (8), (11), and (22) that

$$
\langle E\rangle=-Z^{-1} \frac{\partial Z}{\partial \beta}=-\left\langle\tilde{E}^{\prime}\right\rangle_{\mathrm{SPS}},
$$

where $\langle\mathcal{O}\rangle_{\text {SPS }}$ is defined by eq. (26) and

$$
\tilde{E}^{\prime} \equiv \sum_{k<l} \frac{1}{\omega_{k l}} \frac{\partial \omega_{k l}}{\partial \beta} .
$$

We have used the relation

$$
\frac{\partial}{\partial \beta} \prod_{i<j} \omega_{i j}=\tilde{E}^{\prime} \prod_{i<j} \omega_{i j}
$$

to go from the second line of eq. (B1) to the third. The derivative of $\omega_{k l}$ in the right hand side of eq. (B2) is calculated as

$$
\begin{aligned}
& \frac{\partial \omega_{k l}}{\partial \beta}=\frac{\partial}{\partial \beta}\left\{\delta_{g_{k l}, 0} \mathrm{e}^{-\beta\left[\tilde{V}_{k l}+\Delta V_{k l}^{*}\right]}+\delta_{g_{k l}, 1} \mathrm{e}^{-\beta \bar{V}_{k l}}\right\} \\
& =-\delta_{g_{k l}, 0}\left(\tilde{V}_{k l}+\Delta V_{k l}^{*}\right) \mathrm{e}^{-\beta\left[\tilde{V}_{k l}+\Delta V_{k l}^{*}\right]} \\
& -\delta_{g_{k l}, 1}\left(\bar{V}_{k l}+\beta \frac{\partial \bar{V}_{k l}}{\partial \beta}\right) \mathrm{e}^{-\beta \bar{V}_{k l}} \\
& =\omega_{k l}\left\{-\delta_{g_{k l}, 0}\left(\tilde{V}_{k l}+\Delta V_{k l}^{*}\right)-\delta_{g_{k l}, 1}\left(\bar{V}_{k l}+\beta \frac{\partial \bar{V}_{k l}}{\partial \beta}\right)\right\},
\end{aligned}
$$

where we have used the identity

$$
\begin{aligned}
& A_{k l}^{(0)} B_{k l}^{(0)} \delta_{g_{k l}, 0}+A_{k l}^{(1)} B_{k l}^{(1)} \delta_{g_{k l}, 1} \\
& =\left(A_{k l}^{(0)} \delta_{g_{k l}, 0}+A_{k l}^{(1)} \delta_{g_{k l}, 1}\right)\left(B_{k l}^{(0)} \delta_{g_{k l}, 0}+B_{k l}^{(1)} \delta_{g_{k l}, 1}\right)
\end{aligned}
$$

to go from the third line of eq. (B4) to the fourth. By substituting

$$
\frac{\partial \bar{V}_{k l}}{\partial \beta}=\beta^{-2} \log \left[1-P_{k l}\right]+\beta^{-1} \frac{P_{k l}}{1-P_{k l}}\left(\Delta V_{k l}-\Delta V_{k l}^{*}\right),
$$

and $\delta_{g_{k l}, 0}=1-\delta_{g_{k l}, 1}$ into eq. (B4), we obtain

$$
\frac{\partial \omega_{k l}}{\partial \beta}=\omega_{k l}\left\{-\tilde{V}_{k l}-\Delta V_{k l}^{*}-\delta_{g_{k l}, 1}\left(\frac{\Delta V_{k l}-\Delta V_{k l}^{*}}{1-P_{k l}}\right)\right\}
$$

From this equation and eq. (B2), we find

$$
\tilde{E}^{\prime}=-E_{\text {const }}-\tilde{E},
$$

where $E_{\text {const }}$ and $\tilde{E}$ are defined by eqs. (27) and (28), respectively. Equation (24) is obtained by substituting this equation into eq. (B1).

We next derive a formula for heat capacity $\langle C\rangle$ from eq. (23). To this end, we first calculate the second derivative of $Z$ in the equation. We see from eq. (B3) that

$$
\begin{aligned}
\frac{\partial^{2}}{\partial \beta^{2}} \prod_{i<j} \omega_{i j} & =\frac{\partial}{\partial \beta} \tilde{E}^{\prime} \prod_{i<j} \omega_{i j} \\
& =\left(\tilde{E}^{\prime 2}+\frac{\partial \tilde{E}^{\prime}}{\partial \beta}\right) \prod_{i<j} \omega_{i j}
\end{aligned}
$$

By taking the trace in the both hand slides of the equation, we obtain

$$
\frac{\partial^{2} Z}{\partial \beta^{2}}=Z\left\langle\tilde{E}^{\prime 2}+\frac{\partial \tilde{E}^{\prime}}{\partial \beta}\right\rangle_{\mathrm{SPS}}
$$

Equation (25) is derived from this equation and eqs. (23), (B1), and (B8). Equation (29) is readily obtained from eq. (28). 
[1] A. W. Appel, SIAM J. Sci. Stat. Comput. 6, 85 (1985).

[2] J. Barnes and P. Hut, Nature 324, 446 (1986).

[3] L. Greengard, The Rapid Evolution of Potential Fields in Particle Systems (MIT Press, Cambridge, MA, 1988).

[4] J. Carrier, L. Greengard, and V. Rokhlin, SIAM J. Sci. Stat. Comput. 9, 669 (1988).

[5] M. Saito, Mol. Simul. 8, 321 (1992).

[6] H.-Q. Ding, N. Karasawa, and W. A. Goddard III, J. Chem. Phys. 97, 4309 (1992).

[7] E. Luijten and H. W. J. Blöte, Int. J. Mod. Phys. C 6, 359 (1995).

[8] J. Sasaki and F. Matsubara, J. Phys. Soc. Jpn. 66, 2138 (1996).

[9] B. Hetényi, K. Bernacki, and B. J. Berne, J. Chem. Phys. 117, 8203 (2002).

[10] K. Bernacki, B. Hetényi, and B. J. Berne, J. Chem. Phys. 121, 44 (2004).

[11] M. Sasaki and F. Matsubara, J. Phys. Soc. Jpn. 77, 024004 (2008).

[12] C. H. Mak, J. Chem. Phys. 122, 214110 (2005).

[13] C. H. Mak and A. K. Sharma, Phys. Rev. Lett. 98, 180602 (2007).

[14] When pairwise interactions $V_{i j}$ of an $N$-element system decrease with the distance as $r_{i j}^{-\alpha}$, the number of interac- tions per element switched to $\bar{V}_{i j}$ and computational time per Monte Carlo step are $\mathcal{O}(N)$ for $\alpha>d, \mathcal{O}(N \log N)$ for $\alpha=d$, and $\mathcal{O}\left(N^{2-\alpha / d}\right)$ for $\alpha<d$, where $d$ is the spatial dimension. See ref. [11] for details.

[15] K. Fukui and S. Todo, J. Comp. Phys. 228, 2629 (2009).

[16] P. W. Kasteleyn and C. M. Fortuin, J. Phys. Soc. Jpn. Suppl. 26, 11 (1969).

[17] C. M. Fortuin and P. W. Kasteleyn, Physica 57, 536 (1972).

[18] K. Hukushima and K. Nemoto, J. Phys. Soc. Jpn. 65, 1604 (1996).

[19] R. G. Edwards and A. D. Sokal, Phys. Rev. D 38, 2009 (1988).

[20] K. Matsushita, R. Sugano, A. Kuroda, Y. Tomita, and H. Takayama, J. Phys. Soc. Jpn. 74, 2651 (2005).

[21] S. Romano, Nouvo Cimento D 7, 717 (1986).

[22] H. M.-Krumbhaar and K. Binder, J. Stat. Phys. 8, 1 (1973).

[23] D. P. Landau and K. Binder, A Guide to Monte Carlo Simulations in Statistical Physics, (Cambridge University Press, Cambridge, 2000).

[24] R. H. Swendsen and J.-S. Wang, Phys. Rev. Lett. 58, 86 (1987). 